\title{
Spectral evidence for dust in late-type $M$ dwarfs
}

\author{
Hugh R. A. Jones ${ }^{1}$ and Takashi Tsuji \\ Institute of Astronomy, The University of Tokyo, 2-21-1 Osawa, Mitaka 181, Tokyo, Japan
}

Received __; accepted _

\footnotetext{
${ }^{1}$ Astrophysics Group, Liverpool John Moores University, Byrom St, Liverpool L3 3AF, $\mathrm{UK}$
} 


\begin{abstract}
We have found a dramatic change in the spectra of late-type $M$ dwarfs at wavelengths shorter than $0.75 \mu \mathrm{m}$. Comparisons with synthetic spectra illustrate that this change is well explained by the presence of dust. It thus seems that a substantial part of the long-standing discrepancies between late-type M dwarf models and observations arises from dust as recently proposed by Tsuji, Ohnaka \& Aoki (1996a).

Synthetic spectra and evolutionary models for low-mass objects need to include dust for the reliable modelling of objects below $3000 \mathrm{~K}$.
\end{abstract}

Subject headings: Equation of state - molecular processes - stars: atmospheres: fundamental parameters - stars:late-type - stars: low-mass, brown dwarfs infrared: stars. 


\section{Introduction}

Although the theory of low-mass stars $\left(<0.7 \mathrm{M}_{\odot}\right)$ has a long pedigree (e.g. review Allard \& Hauschildt 1997) models have had relatively little success in matching observed spectral energy distributions. It has long been realised that much of this problem may lie

with the lack of good molecular data. However additional reasons have also been advanced. (1) Brett (1995) propose the neglect of chromospheric hearing and the simple treatment of convection. (2) Jones et al. (1995) propose that the discrepancy with regard to fitting water vapour absorption bands lies in part with the simplistic treatment of water vapour line broadening. (3) Tsuji et al. (1996a) propose that the lack of treatment of dust opacity has been the root of the problems and showed that dusty models explain the photometry of the brown dwarf candidate GD165B (Tsuji et al. 1996b).

Here we present comparisons between M dwarf spectra and the predictions for dusty and non-dusty models. We focus on the region shortward of $1 \mu \mathrm{m}$ where (1) the effects of dust formation can clearly be distinguished from the problems with reliable modelling of water vapour absorption bands and (2) the effects of dust formation will be largest, e.g. fig. 2, Tsuji et al. (1996a).

\section{Observational and synthetic spectra}

Spectra for this paper were taken from a variety of sources allowing us to check that the effects presented did not arise from observational errors. They come from the observations of Kirkpatrick, Henry \& McCarthy (1991), Martin, Rebolo \& Zapatero Osorio (1996) and spectra taken using the FORS and the RGO spectrograph on the AAT. We did find some differences between spectra for objects common to the different observations though these were generally smaller than one spectral type. Apart from the object BRI0021-0214 
(M9.5V) from Martin et al., all the spectra presented are Kirkpatrick et al. spectra and thus should be free of significant differences due to data reduction procedures.

In this paper we discuss the observational spectra in terms of 'spectral type', these exist for large numbers of $\mathrm{M}$ dwarfs, are well quantified and broadly agreed upon. We might have compared spectra in terms of temperature though, the small number of $\mathrm{M}$ dwarfs which have derived temperatures and the large discrepancies (as much as $600 \mathrm{~K}$ ) between the relative scales found by different authors (see discussion Tsuji et al. 1996a) lead us to prefer spectral types. Traditionally spectral types for $\mathrm{M}$ dwarfs were based on relating $\mathrm{TiO}$ and VO band strengths to those used to define types for M giants, though in recent years there has been a move to try to base spectral types on the underlying energy distribution, rather than the strength of any particular feature. The spectral types for the objects presented in this paper are from a least squared minimisation technique (Kirkpatrick et al. 1991) and from indices based on spectral regions of 'psuedocontinuum' (Martin et al. 1996). Based on comparisons with luminosities and colours these measurements are primarily sensitive to temperature. Although there will be some sensitivity to gravity and composition, the objects presented here are generally standards for their particular spectral type and are thus expected to have similar gravities and composition. We thus believe that it is most appropriate to make our comparisons considering spectral type as a surrogate for temperature.

The spectra examined in this paper have been presented a number of times by the above authors and others, however, in Figs 1 and 2 we draw attention to the short-wavelength region $(0.65-0.76 \mu \mathrm{m})$ of the spectra. It can be seen that although the $\mathrm{TiO}$ band strength can be seen to increase between $\mathrm{M} 2 \mathrm{~V}$ and $\mathrm{M} 6.5 \mathrm{~V}$ it dramatically decreases towards later spectral types. This decrease is not expected from dust-free synthetic spectra and represents the essence of what we are highlighting in this paper. 
The models used for this paper were taken from a grid of models computed by Tsuji et al. (1996a) to test the effects of grain formation in low-mass objects. All the synthetic spectra presented here are for solar metallicity, $\log g=5.0$ and a microturbulent velocity of $1 \mathrm{~km} / \mathrm{s}$.

\section{Spectral evidence for dust}

In Fig. 3 we show a spectral sequence made by dividing one standard spectrum by another around two spectral types cooler. The sequence extends from M2V/M4V to $\mathrm{M} 8 \mathrm{~V} / \mathrm{M} 9.5 \mathrm{~V}$. It can be seen that there is a dramatic change in the spectra shortward of $0.75 \mu \mathrm{m}$ around M7V and more subtle changes at longer wavelengths around M8V. This should be compared with the equivalent plot for the synthetic spectra. Fig. 田illustrates that around $2500 \mathrm{~K}$ the models predict changes in spectral characteristics around 0.67 and $0.71 \mu \mathrm{m}$ which are similar in form to Fig. 3 when dust is included in the models. The feature at $0.80 \mu \mathrm{m}$ is not well reproduced and is due to a lack of reliable data for VO whose influence is overestimated by the models (e.g. fig. 12, Brett 1995).

We have investigated dust-free models from 1000-3800 K with metallicities ranging from solar to -2 dex and solar metallicity dusty models from 1000-2700 K. We have not investigated the effect of changing gravity on the models, however based on the work of Jones et al. (1996) we expect that gravity differences will be small. Although there are changes in the characteristics of the spectra due to temperature (around 1500 and 2300 K) and metallicity we have not found any combinations of hotter/cooler pairs of dust-free models which are close to reproducing the observations. We have only been able to model the change in spectral characteristics around M7V by the inclusion of dust.

In Fig. 5 we show direct comparisons between observational and model spectra 
from M6V to M9V. It can be seen that observational features are reasonably matched by dusty models. Given the preliminary treatment of dust employed and the uncertainties in the molecular data the fit is a lot better than we might expect. The observations might also be explained by a combination of inadequacies in our models: unaccounted for absorption bands between the $\mathrm{TiO}$ and $\mathrm{VO}$ bands, the presence of a temperature minimum, a chromosphere and an overly simplistic treatment of the molecular opacities. Although it is important to investigate these uncertainties we favour an explanation which is substantially due to dust because with a classical treatment of dust it is possible to explain the long-standing problem of fitting the observed water bands (Tsuji et al. 1996a) and at the same time elegantly explain the behaviour of the short wavelength spectra.

\section{Discussion}

The implication of this work is that dust needs to be considered in models of objects below $3000 \mathrm{~K}$. This is necessary to enable a better fit with observed spectra and photometry, for the derivation of evolutionary tracks for objects below $3000 \mathrm{~K}$ and to find a reliable value for the hydrogen-burning limit. In addition we caution against the use of specific molecular bands for assigning spectral types in late-type M stars.

Given that a dusty model gives a reasonable explanation of observed spectral differences in late-type M dwarfs further observations should be made (or possibly dearchived). Spectra for a range of dwarfs and giants would enable the macroscopic properties of dust to be constrained at pressures differing by around $10^{6}$ across a range of temperature of perhaps 1800-3000 K. We suggest a number of observational programmes to constrain dust.

- The models employed in this paper include dust grains of $\mathrm{Al}_{2} \mathrm{O}_{3}$ (corundum), $\mathrm{Fe}$ (iron) and $\mathrm{MgSiO}_{3}$ (enstatite). Based on fig. 1 (Tsuji et al. 1996a), $\mathrm{Al}_{2} \mathrm{O}_{3}$ dust grains 
are responsible for most of the changes seen in the spectra presented here. By careful observations of atomic $\mathrm{Al}$ and $\mathrm{Mg}$ lines across the transition from dust-free to dusty spectra it should be possible to place constraints on the dust mass and thus the efficiency of dust formation as a function of temperature, pressure and abundance.

- Spectra could be investigated for variability due to dust absorption thereby constraining the presence of dust clouds in late-type objects. Based on the spectra that we have available for vB10 we do find a significant difference between them. However to reliably test this hypothesis we feel that it is necessary to use data taken using the same observational setup and data reduction procedures.

- Spectra at shorter wavelengths than investigated here are expected to have a stronger dust signature and thus can thus yield an empirical high temperature limit to $\mathrm{Al}_{2} \mathrm{O}_{3}$ dust formation.

Such tests are not only vital for understanding the spectral properties of late-type dwarfs stars sufficiently to determine masses but also to constrain whether radiation pressure on dust is sufficient to expel matter directly from the photosphere and is thus the underlying cause of stellar mass-loss in cool giants (e.g. Tsuji 1996).

It is a pleasure to thank Davy Kirkpatrick and Eduardo Martin for generously sharing their data. HRAJ acknowledges PPARC for travel funds. The work was carried out whilst HRAJ was an EU/JSPS Research Fellow. 


\section{REFERENCES}

Allard F., Hauschildt P., 1997, ARAA, in press

Brett J. M., 1995, A\&A, 295, 736

Jones H.R.A., Longmore A.J., Allard F., Hauschildt P.H., Miller S., Tennyson J., 1995, MNRAS, 277, 767

Jones H.R.A., Longmore A.J., Allard F., Hauschildt P.H., 1996, MNRAS, 280, 77

Kirkpatrick J.D., Henry T.J., McCarthy D.W., 1991, ApJS, 77, 417

Martin E.L., Rebolo R., Zapatero-Osorio M. R., 1996, ApJ, 469, 706

Tsuji T., Ohnaka K., Aoki W., 1996a, A\&A, 305, L1

Tsuji T., Ohnaka K., Aoki W., Nakajima T., 1996b, A\&A, 308, L29

Tsuji T. 1996, IAU Symp. 177, in press, ed. Wing R. F. 
Fig. 1.- Spectra of Gl411 (M2V), Gl268ab (M4.5V) and LHS523 (M6.5V) are overlayed with their flux normalised to be one at $0.758 \mu \mathrm{m}$. The deep absorption bands centered around 0.68 and $0.71 \mu \mathrm{m}$ increasing with spectral type are due to TiO.

Fig. 2.- Spectra of LHS523 (M6.5V), vB10 (M8V) and BRI0021-0214 (M9.5V) are overlayed with their flux normalised to be one at $0.758 \mu \mathrm{m}$.

Fig. 3.- A sequence of 'divided' spectra which have been offset from one another. Working from top down, the uppermost spectra represents $\mathrm{M} 2 \mathrm{~V} / \mathrm{M} 4.5 \mathrm{~V}$ (Gl411/Gl268ab), then M4.5V/M6V (Gl268ab/Gl406), M6V/M8V (Gl406/vB10) and M8V/M9.5V (vB10/BRI0021-0214).

Fig. 4.- Both spectra are a hotter model divided by a cooler one in the same fashion as the observations in Fig. 3. In both cases the hotter model is a $2600 \mathrm{~K}$ dust-free model; in the upper plot the cooler model is a $2400 \mathrm{~K}$ dust-free model; in the lower plot the cooler model is a $2400 \mathrm{~K}$ dusty model.

Fig. 5.- A comparison between observed spectra (solid), dusty models (dashed) and dustfree models (dotted): (a) Gl406(M6V) and $2600 \mathrm{~K}$ models, (b) vB10 (M8V) and $2200 \mathrm{~K}$ models, (c) LHS2924 (M9V) and $2000 \mathrm{~K}$ models. 


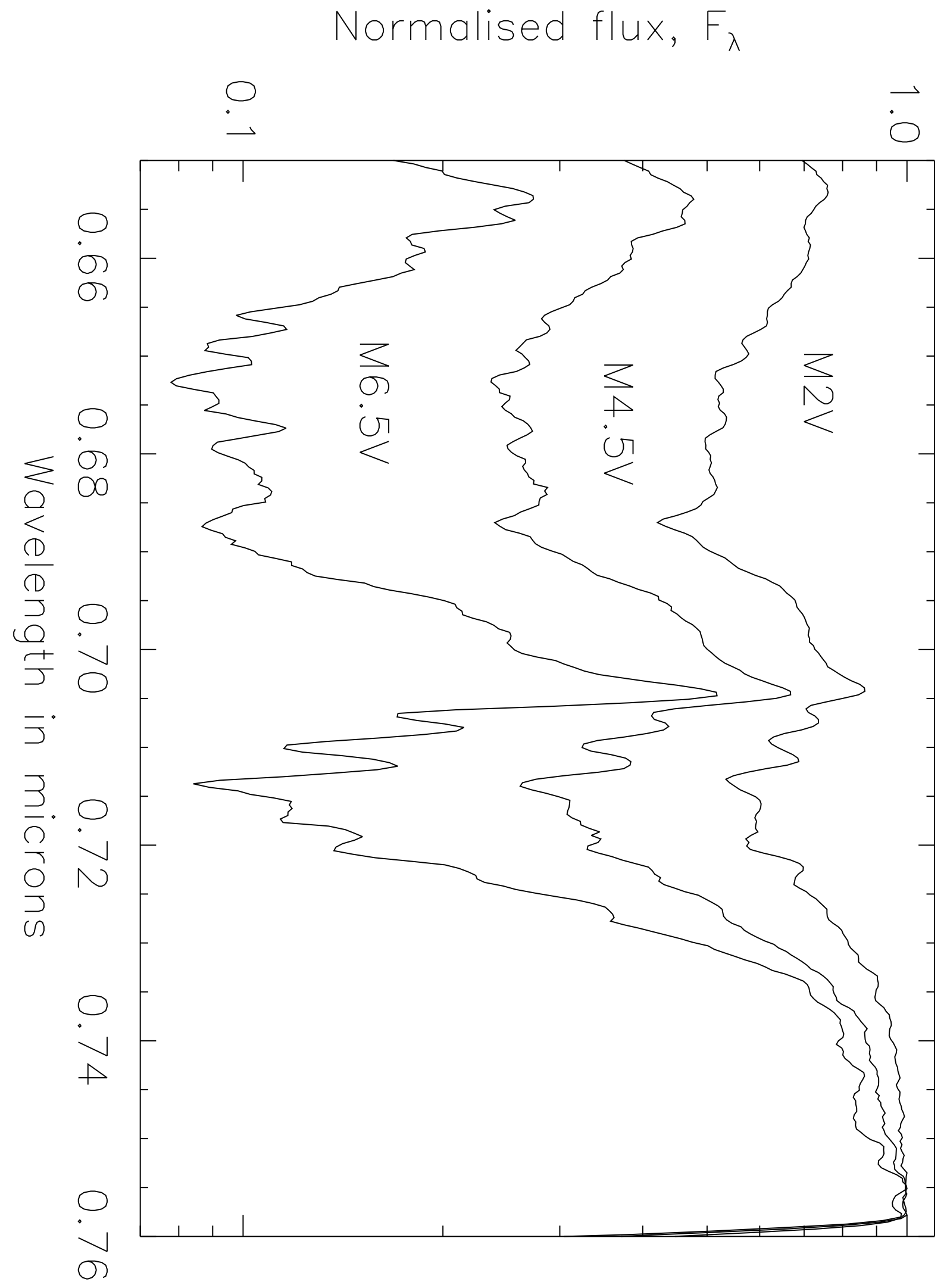




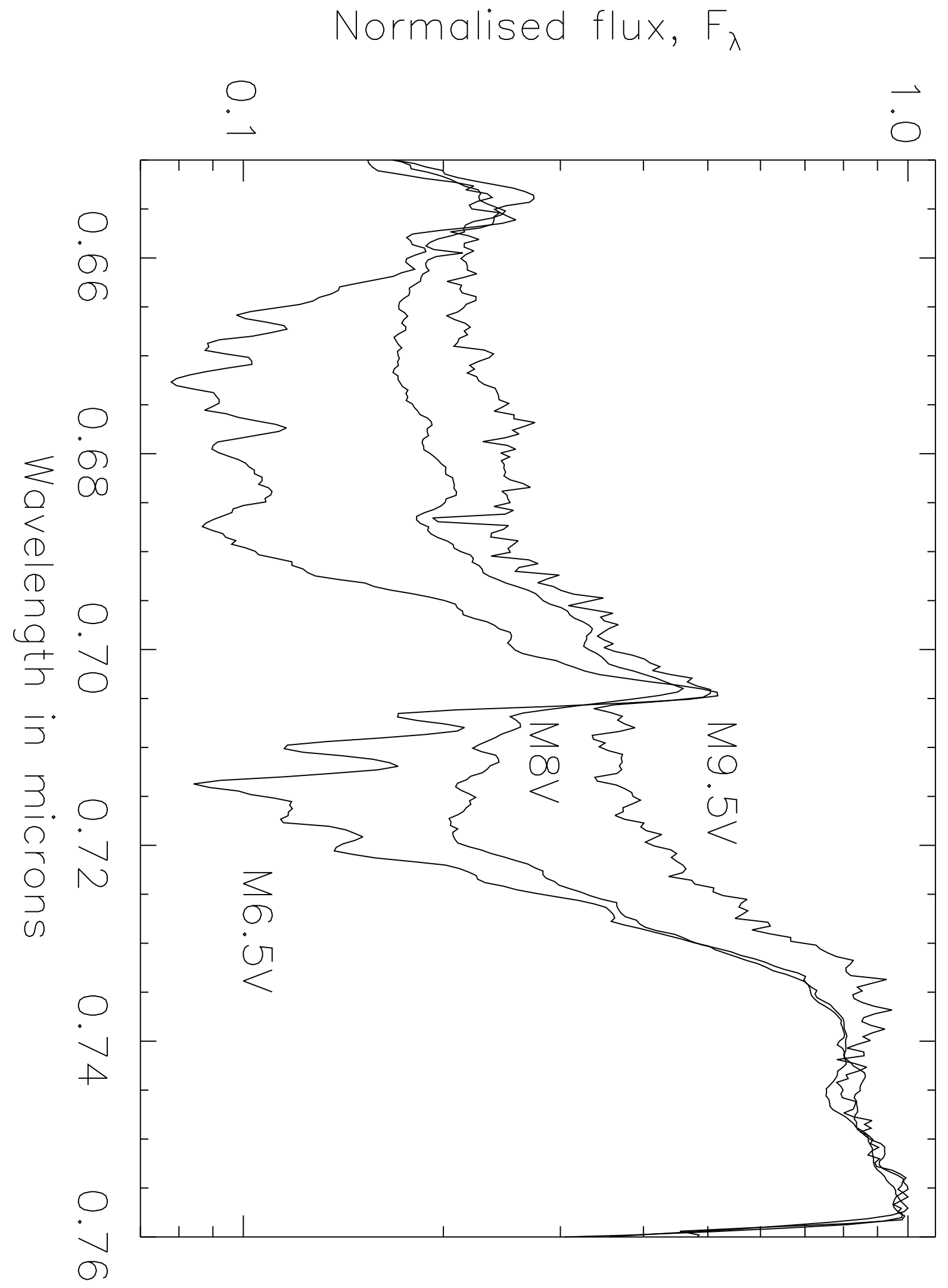




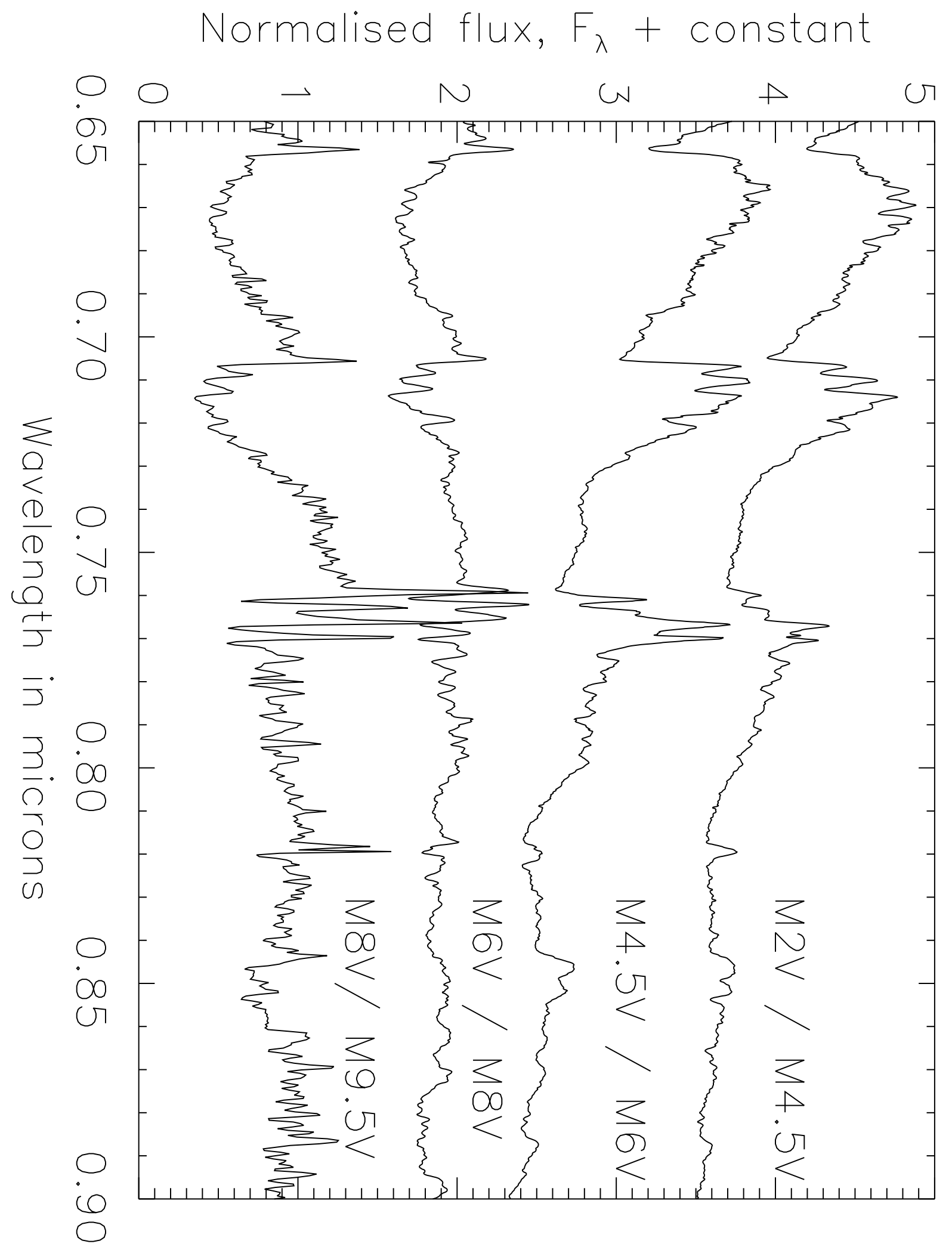




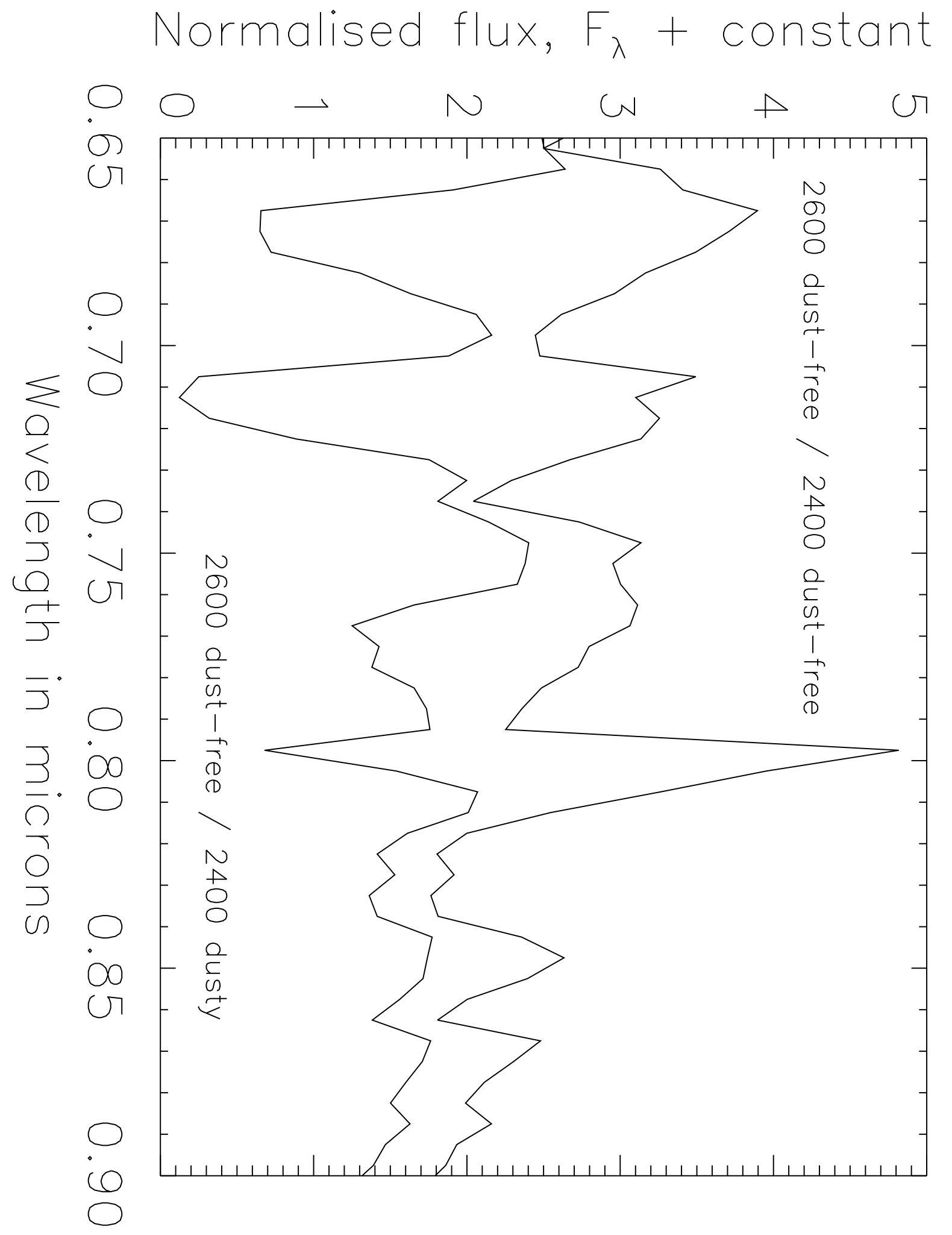




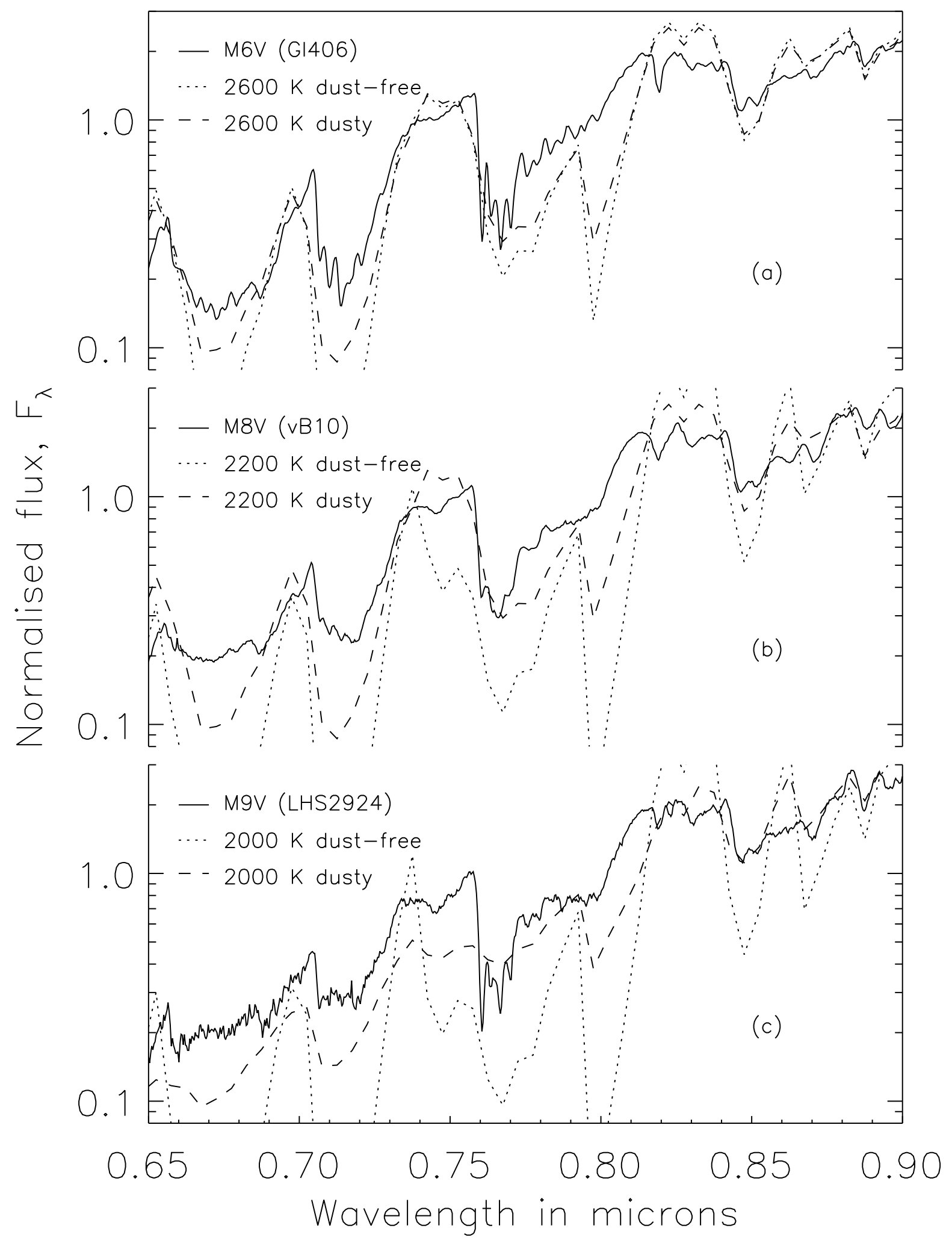

